\title{
Genetic analysis of post-mating reproductive barriers in hybridizing European Populus species
}

\author{
D Macaya-Sanz ${ }^{1}$, L Suter ${ }^{2,3}$, J Joseph ${ }^{2}$, T Barbará2,4, N Alba ${ }^{1}$, SC González-Martínez ${ }^{1}$, A Widmer ${ }^{3}$ \\ and C Lexer ${ }^{2,4}$ \\ ${ }^{1}$ Department of Forest Ecology and Genetics, Center of Forest Research, CIFOR-INIA, Carretera de A Coruña, Madrid, Spain; \\ ${ }^{2} J o d r e l l$ Laboratory, Royal Botanic Gardens, Kew, Richmond, Surrey, UK; ${ }^{3} E T H$ Zürich, Institute of Integrative Biologie, \\ Universitätstrasse 16, Zürich, Switzerland and ${ }^{4}$ Department of Biology, University of Fribourg, Unit of Ecology and Evolution, \\ Fribourg, Switzerland
}

\begin{abstract}
Molecular genetic analyses of experimental crosses provide important information on the strength and nature of postmating barriers to gene exchange between divergent populations, which are topics of great interest to evolutionary geneticists and breeders. Although not a trivial task in longlived organisms such as trees, experimental interspecific recombinants can sometimes be created through controlled crosses involving natural $F_{1}$ 's. Here, we used this approach to understand the genetics of post-mating isolation and barriers to introgression in Populus alba and Populus tremula, two ecologically divergent, hybridizing forest trees. We studied 86 interspecific backcross $\left(\mathrm{BC}_{1}\right)$ progeny and $>350$ individuals from natural populations of these species for up to 98 nuclear genetic markers, including microsatellites, indels and single nucleotide polymorphisms, and inferred the origin of the cytoplasm of the cross with plastid DNA. Genetic analysis of the $\mathrm{BC}_{1}$ revealed extensive
\end{abstract}

segregation distortions on six chromosomes, and $>90 \%$ of these (12 out of 13) favored $P$. tremula donor alleles in the heterospecific genomic background. Since selection was documented during early diploid stages of the progeny, this surprising result was attributed to epistasis, cyto-nuclear coadaptation, heterozygote advantage at nuclear loci experiencing introgression or a combination of these. Our results indicate that gene flow across 'porous' species barriers affects these poplars and aspens beyond neutral, Mendelian expectations and suggests the mechanisms responsible. Contrary to expectations, the Populus sex determination region is not protected from introgression. Understanding the population dynamics of the Populus sex determination region will require tests based on natural interspecific hybrid zones.

Heredity (2011) 107, 478-486; doi:10.1038/hdy.2011.35; published online 18 May 2011

Keywords: speciation; hybrid zone; Populus; introgression; segregation distortion; sex chromosome

\section{Introduction}

The genetics of reproductive barriers is of great current interest in evolutionary genetics, because the extent of gene flow experienced by diverging populations or species depends crucially on it (Coyne and Orr, 2004). Reproductive isolation (RI) is a key feature of Mayr's Biological Species Concept, which includes the notion of whole genome isolation between divergent taxa as a hallmark of 'good' species. More recently, a 'genic view' of species and speciation has found wide acceptance, which recognizes that genomes can be porous and that RI is a property of individual loci or genomic regions, rather than the genome as a whole (Wu, 2001; Lexer and Widmer, 2008).

RI will first arise in genomic regions harboring 'speciation genes' or other isolation factors, leading to genomic islands of divergence (Wu, 2001; Emelianov et al., 2004; Nosil et al., 2009), and will subsequently spread across the genome. Nevertheless, many groups

Correspondence: Professor C Lexer, Department of Biology, Unit of Ecology and Evolution, University of Fribourg, Chemin du Musée 10, Fribourg, CH 1700, Switzerland.

E-mail: christian.lexer@unifr.ch

Received 20 October 2010; revised 16 March 2011; accepted 31

March 2011; published online 18 May 2011 of taxa do not achieve complete genomic isolation for millions of years, as observed in Helianthus (sunflowers), Populus (poplars, aspen, cottonwoods), Quercus (oaks), Silene (campions) or Iris (lilies), to name just a few examples among plants (reviewed by Lexer and Widmer, 2008). Despite the shift in perception from whole genome isolation to a genic view of species, the genetics of RI is still at the center of attention in speciation genetics (Widmer et al., 2009). This is the case because the genetics of RI will determine how quickly gene flow ceases and which loci are affected first.

Plant speciation geneticists often use controlled crosses to study the genetics of post-mating components of RI (Fishman et al., 2001; Coyne and Orr, 2004; Bouck et al., 2005; Sweigart et al., 2006). Even in species with strong reproductive barriers, interspecific multi-generation crosses can sometimes be obtained in the laboratory. In such crosses, interspecific recombination in the meiosis of the $F_{1}$ will effectively start to break up the parental species' genomes, which allows geneticists to isolate and study chromosomal blocks with a role in moderating gene flow (Lexer and Widmer, 2008). One approach to achieve this goal is to search for loci or genomic regions with departures from Mendelian expectations, also known as segregation distortion (Fishman et al., 2001; Yin et al., 2004; Bouck et al., 2005; Sweigart et al., 2006; 
Lopez-Fernandez and Bolnick, 2007). As expected from theory (Barton, 2001; Wu, 2001; Turelli and Moyle, 2007), such studies tend to recover the full breadth of departures from Mendelian expectations, including genome regions that cause isolation and resist introgression (Fishman et al., 2001; Sweigart et al., 2006), and loci that cross the barrier more readily than expected based on Mendel's laws (Tiffin et al., 2001; Yin et al., 2004; Bouck et al., 2005).

Experimental cross-based approaches such as this (beyond the $F_{1}$ ) have rarely been used in forest trees because of the difficulty of creating recombinant hybrid generations in species with long generation times (but see Yin et al., 2004; Yin et al., 2008). The scarcity of experimental multi-generation crosses in forest trees is unfortunate, as long-lived trees provide the opportunity to dissect post-mating barriers in organisms with juvenile-adult phase change, that is potentially from gametes through early embryonic and juvenile stages to maturity. One way to circumvent the long time needed to generate experimental crosses in forest trees is to take advantage of natural hybrids (for example Woolbright et al., 2008), but this approach remains largely unexplored.

Populus alba and Populus tremula are two ecologically divergent European members of the 'model tree' genus Populus. Reproductive barriers between these species are incomplete, leading to the frequent formation of extensive 'mosaic' hybrid zones (Lexer et al., 2005, 2010). Ongoing genomic studies of natural populations indicate that the species boundary is porous, with some loci resisting introgression and others crossing the barrier more readily than predicted by genomic expectations (Lexer et al., 2007, 2010). Morphometric data indicate introgression of phenotypic traits from $P$. tremula into $P$. alba (Lexer et al., 2009), despite the presence of substantial RI involving both assortative mating and post-zygotic isolation in the form of epistatic interactions (Lexer et al., 2010). Studies of controlled interspecific progeny are useful for reducing the complexity of patterns of RI seen in situ in natural hybrid zones.

Since $P$. alba and $P$. tremula are dioecious and sex determination in Populus appears to be controlled by an incipient sex chromosome (Yin et al., 2008; Pakull et al., 2009; Paolucci et al., 2010), controlled crosses also provide the opportunity to study the role of the sex determination region in blocking interspecific gene flow, which is a topic of great current interest in evolutionary genetics (Qvarnström and Bailey, 2009). Our hypothesis at the outset of this study was that species isolation genes will have accumulated in the sex determination region of poplar, as recently observed for other taxa (Qvarnström and Bailey, 2009).

With this in mind, the questions of this study were as follows: (1) What do marker segregation data from a controlled interspecific backcross $\left(\mathrm{BC}_{1}\right)$ tell us about the strength and genetic architecture of post-mating reproductive barriers in these hybridizing forest trees? (2) How great is the potential for interspecific introgression across porous species boundaries in Populus? (3) What is the likely role of the Populus sex determination region in blocking or moderating interspecific gene flow? To address these questions, we analyzed and interpreted segregation patterns of alleles of known species origin for a genome-wide panel of molecular genetic markers, genotyped in a controlled interspecific $\mathrm{BC}_{1}$ of $P$. alba and $P$. tremula, and compared the results to patterns of divergence and linkage disequilibrium (LD) in natural populations.

\section{Materials and methods}

\section{Plant materials $\left(\mathrm{BC}_{1}\right.$ and natural populations)}

To study post-mating reproductive barriers creating segregation distortions, we developed a controlled backcross of an $\mathrm{F}_{1}$ natural poplar hybrid (Populus tremula $\times$ $a l b a)$ with a pure $P$. alba $\left(\mathrm{BC}_{1}\right)$. The male parent of this cross was a known natural clone (J1) of P. alba from the Jalón river in the Ebro watershed (Northeast of the Iberian Peninsula), and the female parent an $\mathrm{F}_{1}$ natural hybrid (BET3) from a hybrid population in the Tajo river headwaters (Central Iberian Peninsula). The $F_{1}$ hybrid status of BET3 was assessed through phenotypic features and confirmed prior to this study by genomic admixture analysis in STRUCTURE 2.2 following Lexer et al., 2005 (95\% credible intervals of admixture coefficient $Q$ did not include 0.25 or 0.75 ). The species origin of maternally inherited plastid DNA in BET3 was identified here by sequencing the trnC-petN1 plastid DNA region in this interspecific $F_{1}$ hybrid and comparison with sequences from pure individuals of $P$. alba and $P$. tremula; plastid DNA haplotypes are known to be highly divergent between $P$. alba and P. tremula (Lexer et al., 2005; Fussi et al., 2010).

The controlled backcross was produced at INIA's nursery (Madrid, Spain), yielding 131 seedlings that were further grown under greenhouse conditions. Early mortality in first-year seedlings reduced the number of offspring available for DNA extraction and genotyping to 86 individuals, currently maintained in two clone banks planted in Central Spain.

Apart from the $\mathrm{BC}_{1}$ used for mapping, up to 201 individuals from European populations of $P$. tremula and up to 167 individuals from European populations of $P$. alba were employed to determine the parental species origin of markers used in segregation analysis, and for additional population genetic analysis of markers located on chromosome XIX (below). Population genetic data for this purpose were taken from de Carvalho et al. (2010) and Lexer et al. (2010), where detailed documentation of populations and genotypic data can be found. Briefly, populations of P. tremula were from Spain, Scotland, Central Sweden and Austria (two populations, one from the Eastern Alps and one from the Bohemian Massif). Populations of $P$. alba were from Spain, the Austrian Danube and the Hungarian Tisza valley. In addition, 20 individuals of $P$. alba whose sex had been determined phenotypically during the flowering season were sampled in Spain (12 females and 8 males). Species assignment for each individual was achieved using STRUCTURE 2.2 as described above.

\section{Molecular genetic markers and genotyping reactions} Genomic DNA was purified from young fresh leaves using the DNeasy Plant Mini kit (QIAGEN, Hilden, Germany). A genome-wide set of 98 nuclear markers was used for segregation analysis in the interspecific $\mathrm{BC}_{1}$ (Supplementary Tables 1 and 4). These markers included microsatellite loci available from the Populus genome 
consortium (Van der Schoot et al., 2000; Smulders et al., 2001; Tuskan et al., 2006; Yin et al., 2009), microsatellites isolated de novo by our group from expressed sequence tags and from genomic sequence for contig 117 of Populus trichocarpa genome assembly v.1, homologous to chromosome XIX of Populus (Joseph and Lexer, 2008; de Carvalho et al., 2010), and single nucleotide polymorphisms as well as insertion-deletion (indel) markers isolated from expressed sequence tags representing candidate genes for traits involved in ecological divergence between $P$. alba and $P$. tremula (Joseph and Lexer, 2008). The trnC-petN1 plastid DNA region was sequenced in the parents of the mapping cross using essentially the same protocols. All of the marker loci are reported and documented in detail elsewhere (Joseph and Lexer 2008; de Carvalho et al., 2010; Lexer et al., 2010), with the exception of a small number of new microsatellites from chromosome XIX, the incipient sex chromosome of Populus. Because of their special relevance to the objectives of this paper, all chromosome XIX loci are documented once more in detail in the supporting materials of the present paper (Supplementary Table 2).

Forward primers for all nuclear microsatellite markers (86 loci in total; Supplementary Table 1) were M13-tailed, and standard polymerase chain reaction protocols were used for DNA amplification following Lexer et al. (2005). Allele sizes were resolved using an Applied Biosystems (ABI) 3100 Genetic Analyzer (Applied Biosystems, Carlsbad, CA, USA) and FAM and JOE fluorescent dyes. Indel polymorphisms in five expressed sequence tags (Joseph and Lexer, 2008) were genotyped as length polymorphisms, using the same methods. For seven further expressed sequence tags (Supplementary Table 1 ), single nucleotide polymorphisms were identified by resequencing the parents of the $\mathrm{BC}_{1}$ cross following Joseph and Lexer (2008), and the progeny was genotyped for each single nucleotide polymorphism using the ABI $\mathrm{SNaPshot}$ assay (Applied Biosystems) following the manufacturer's instructions.

\section{Data analysis}

Segregation analysis in the interspecific $\mathrm{BC}_{1}$ : Marker alleles segregating from the interspecific $F_{1}$ hybrid parent BET3 were analyzed in the interspecific $\mathrm{BC}_{1}$ progeny. The focus of this study was on patterns of single marker segregation-linkage analysis was only used as a quality control (below). Both segregation and linkage analyses were carried out in MAPMANAGER QTX. This program uses $\chi^{2}$ statistics to test for deviations of marker segregation from Mendelian expectations, equivalent to tests for gametic or allelic segregation distortion. In addition, tests for distortions of particular genotypic classes (=zygotic distortions) were carried out using $\chi^{2}$ tests in JOINMAP 3.0.

Species origin of the hybrid parent (BET3) alleles: The parental species frequencies of alleles found in the $F_{1}$ hybrid parent (BET3) were estimated from natural populations of $P$. alba and P. tremula (see above-Plant materials). Estimating parental frequencies was possible despite the somewhat heterogenous geographic sampling of the parental species (above), because an overwhelming proportion of molecular variance in these European poplars and aspens resides between species, rather than between populations of the same species (Lexer et al., 2005; de Carvalho et al., 2010).

The odds-ratio tests were based on a contingency table constructed using the frequency of each allele in the two species, using proc FREQ in SAS version 9 (SAS Institute Inc, Cary, NC, USA). When the allele frequency was zero or one in any of the species, odds ratios were undefined. In that case, a Fisher's exact test was performed to test for significant differences in the contingency table. For a small number of loci for which the odds-ratio test was not significant, species origin was inferred based on linkage with markers with clearly assigned species origin (Supplementary Table 1; species origin for these markers is given in parentheses). Information about the species origin of each allele in the BET3 hybrid parent was used to determine the direction of segregation distortions in the interspecific $\mathrm{BC}_{1}$, either toward $P$. alba or toward $P$. tremula.

Synteny with $P$. trichocarpa: Although the main objective of this paper was to study single marker segregation distortions, marker coverage on several chromosomes was suitable for defining linkage groups. This was the case because these markers had been picked to tag clusters of quantitative trait loci-controlling species differences in a related project on admixture mapping in Populus (Lexer et al., 2010). These markers provided a useful quality control for our segregation data, as they allowed us to assess levels of synteny of these linkage groups between our European Populus species and the sequenced genome of $P$. trichocarpa.

To examine synteny, linkage groups in the interspecific $B_{1}$ were determined based on log-of-odds likelihood statistics calculated by MAPMANAGER QTX. Marker groupings detected at a log-of-odd threshold of 3.00 were ordered locally by a multi-point analysis using the 'Ripple' command, and map distances in centimorgans were estimated from recombination frequencies using the Kosambi mapping function. Synteny between marker order in the interspecific $\mathrm{BC}_{1}$ and $P$. trichocarpa was evaluated using $P$. trichocarpa genome assembly v2 (available at http://www.phytozome.net/poplar). Physical positions of all markers in $P$. trichocarpa were determined using blast-n searches of the primer sequences against $P$. trichocarpa genome assembly v2, using an $E$-value threshold of 0.1 .

Analysis of chromosome XIX in natural populations: Markers located on the putative sex chromosome XIX were of special interest to this study, because sex chromosomes are often seen as 'hotspots' for species isolation genes. Analyzing natural populations places greater demands on the ease of scoring and robustness of molecular markers than segregation analysis in a simple, controlled pedigree; eight out of nine microsatellites from chromosome XIX (Supplementary Table 2) yielded readily interpretable, fully codominant marker genotypes in the 20 individuals of known sex sampled from natural populations of $P$. alba in Spain (Table 2), and six loci did so across European populations of both species, $P$. alba and P. tremula (3; Supplementary Table 3).

The loci were characterized via the number of alleles $(A)$, expected $\left(H_{\mathrm{E}}\right)$ and observed $\left(H_{\mathrm{O}}\right)$ heterozygosity and within-population inbreeding coefficients $\left(F_{\mathrm{IS}}\right)$ in populations using the computer program FSTAT (Goudet, 
1995). LD between pairs of loci was estimated using the common marker correlation and exact $P$-values for LD were computed with GENEPOP (Rousset, 2008). To keep the number of pair wise LD tests manageable, these analyses were restricted to four populations of particular interest to ongoing evolutionary genetics research in these species: Danube (Austria) and Tisza (Hungary) for P. alba, and Eastern Alps (Austria) and Central Sweden for $P$. tremula. In addition, the 20 Spanish individuals of known sex were characterized for their diversity and heterozygosity $\left(H_{\mathrm{E}}\right.$ and $\left.H_{\mathrm{O}}\right)$.

To yield insights into the role of the putative sex chromosome XIX in blocking gene flow between $P$. alba and $P$. tremula, interspecific genetic divergence for chromosome XIX markers was estimated in the form of $F_{\mathrm{ST}}$ and the results confirmed by $G_{S T}^{\prime}$, a standardized differentiation measure that takes within-population heterozygosity into account (Hedrick, 2005). To relate interspecific divergence on chromosome XIX to the genome-wide average, $F_{\mathrm{ST}}$ values for chromosome XIX were compared with genomewide expectations for 93 microsatellite- and sequence-based genetic markers reported by Lexer et al. (2010).

\section{Results}

Marker polymorphism and species origin of donor alleles Ninety-eight (98) genetic markers (microsatellites, indels and single nucleotide polymorphisms) representing all 19 chromosomes of the Populus genome were analyzed in the interspecific $\mathrm{BC}_{1}$ of $P$. alba and P. tremula (Supplementary Tables 1 and 4). Out of these, 39 (40\%) were polymorphic in the female $\mathrm{F}_{1}$ hybrid parent (BET3) only, $11(11 \%)$ were polymorphic in the male P. alba backcross parent (J1) only and $26(27 \%)$ were polymorphic in both parents, whereas $22(22 \%)$ were monomorphic in both parents of the cross (Supplementary Table 1). The oddsratio test and Fisher's exact test facilitated statistical assignment of species origin of alleles segregating from the female $F_{1}$ hybrid parent (BET3) for 47 of the markers. For 13 further loci, putative species origin of alleles segregating from the $\mathrm{F}_{1}$ hybrid parent could be assigned based on linkage to markers with clear species assignments (Supplementary Table 1). With respect to organellar DNA, plastid DNA sequencing revealed a $P$. tremula haplotype for BET3, thus indicating the species origin of the cytoplasm of the interspecific $\mathrm{BC}_{1}$ cross.

\section{Segregation distortions}

Thirteen markers on six chromosomes (20\% of polymorphic markers) displayed significant segregation distortion of alleles segregating from the BET3 hybrid parent of the $\mathrm{BC}_{1}$ (Table 1), compared with three markers expected by chance alone. All of these loci displayed genotypic (= zygotic) segregation distortion as well. For 12 of these loci, the $P$. tremula (=donor) allele was significantly overrepresented in the backcross progeny. Only for 1 of the 13 distorted markers, segregation distortion was against the introgressed $P$. tremula allele (that is the $P$. alba allele segregating from the $F_{1}$ hybrid parent was overrepresented instead; Table 1). The results allowed us to discuss the strength of post-mating reproductive barriers between these hybridizing species (see below).

\section{Synteny with $P$. trichocarpa}

All detected linkages were conserved between $P$. trichocarpa genome assembly v. 2 and the present interspecific $\mathrm{BC}_{1}$ of $P$. alba and P. tremula (Supplementary Table 1). Synteny is best exemplified by chromosome VI, known to exhibit normal levels of recombination (Yin et al., 2004), and chromosome XIX, known to exhibit suppressed recombination (Yin et al., 2008) (Figure 1). Marker order was completely conserved on chromosome VI, whereas no recombination event was observed between four markers on chromosome XIX in the interspecific $\mathrm{BC}_{1}$, corresponding to $>560 \mathrm{~kb}$ on the $P$. trichocarpa physical genome map (Figure 1; see also below).

Segregation distortion and diversity of chromosome XIX Three loci on the proximal end of chromosome XIX, the incipient sex chromosome of Populus, displayed segregation distortion in the female $F_{1}$ hybrid parent (BET3) in the form of an overrepresentation of donor alleles from

Table 1 Genetic markers with segregation distortion in an interspecific $\mathrm{BC}_{1}$ between $P$. tremula and $P$. alba, including chromosome assignment on $P$. trichocarpa genome assembly v.2, significance levels of segregation distortions in the $\mathrm{BC}_{1}$, identity of the over-represented allele for each locus, odds ratios for parental species assignments of alleles in natural populations and inferred species assignment of the overrepresented allele

\begin{tabular}{|c|c|c|c|c|c|c|}
\hline Locus & $\begin{array}{l}\text { P. trichocarpa } \\
\text { chromosome }\end{array}$ & $\begin{array}{l}\text { Distortion } P . \text { alba } \times \\
\text { tremula F1 parent }(\stackrel{+}{)})\end{array}$ & $\begin{array}{l}\text { Overrepresented } \\
\text { allele }\end{array}$ & $\begin{array}{c}\text { Odds ratio } P \text {. albal } \\
\text { tremula } F 1(q) \text { allele } 1\end{array}$ & $\begin{array}{c}\text { Odds ratio } P \text {. albal } \\
\text { tremula } F 1(q) \text { allele } 2\end{array}$ & $\begin{array}{c}\text { Species origin of overrepresented } \\
\text { allele from } F 1\left(\begin{array}{l}(\mathrm{q})\end{array}\right.\end{array}$ \\
\hline GCPM 1274 & 1 & $* * * *$ & 2 & $1.55 / 0.45$ & $0.93 / 1.32$ & (P. tremula) \\
\hline ASP 112376 & 1 & $*$ & 1 & $3.18 / 0.05$ & $0.00 / 3.34$ & P. alba \\
\hline GCPM 124 & 1 & $* *$ & 2 & $42.20 / 0.07$ & $0.11 / 2.90$ & P. tremula \\
\hline GCPM 1629 & 3 & $* * * * *$ & 1 & $0.62 / 5.02$ & $1.24 / 0.00$ & P. tremula \\
\hline Thau & 9 & $* *$ & 2 & $2.44 / 0.00$ & NA & (P. tremula) \\
\hline ORPM 23 & 9 & $* * * * *$ & 1 & $0.00 / 3.49$ & $1.61 / 0.00$ & P. tremula \\
\hline ORNL 149 & 10 & * & 1 & $0.00 / 3.8$ & $14.00 / 0.00$ & P. tremula \\
\hline ORPM 344 & 10 & $* * * *$ & 1 & $0.00 / 3.73$ & $2.27 / 0.25$ & P. tremula \\
\hline GCPM 1250 & 10 & $* * * *$ & 2 & $4.03 / 0.04$ & $0.25 / 23.05$ & P. tremula \\
\hline GCPM 154 & 12 & $* * * * *$ & 1 & $0.00 / 2.79$ & NA & (P. tremula) \\
\hline Yin1 & 19 & $* *$ & 1 & NA & $1.28 / 0.73$ & P. tremula \\
\hline Yin2 & 19 & $*$ & 2 & $2.64 / 0.00$ & $0.00 / 1.90$ & P. tremula \\
\hline ORPM 206 & 19 & $* * *$ & 1 & $0.00 / 4.06$ & $10.27 / 0.00$ & P. tremula \\
\hline
\end{tabular}

Abbreviations: BC, backcross; NA, not applicable.

Species assignments supported by the genotype data, but not significant in the odds-ratio test are shown in parentheses. Significance thresholds from $\chi^{2}$ tests.

${ }^{*} P<0.05$, ${ }^{* *} P<0.01$, ${ }^{* * *} P<0.005$, ${ }^{* * * *} P<0.001$, ***** $P<0.00005$. 

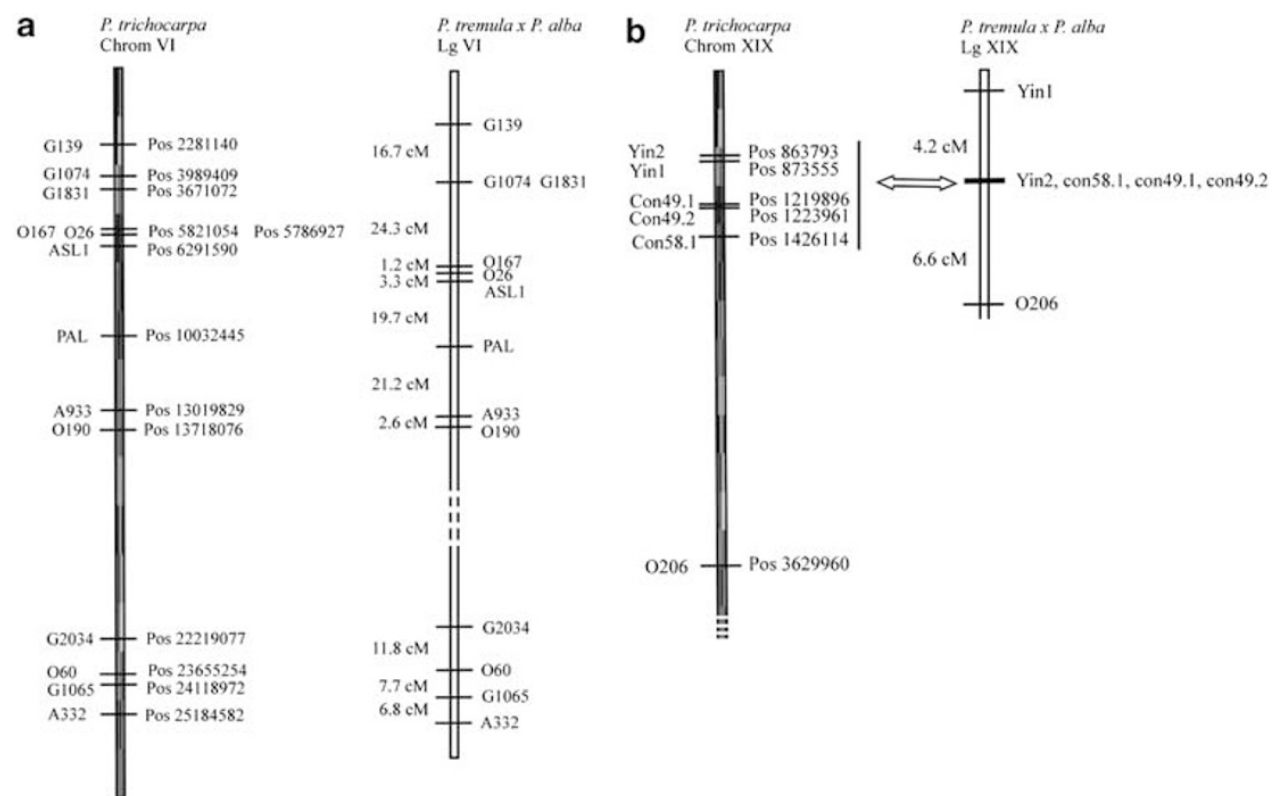

Figure 1 Comparison of the P. tremula $\times$ P. alba linkage map to P. trichocarpa genome assembly v.2 for chromosome VI (a), known to exhibit normal levels of recombination (Yin et al., 2004), and chromosome XIX (b), known to exhibit greatly reduced recombination (Yin et al., 2008). Complete synteny between the two maps is indicated by the conserved marker order on chromosome VI. On chromosome XIX, zero recombination was observed between markers Yin2, con58.1, con49.1 and con49.2 on the P. tremula $\times$ P. alba linkage map (indicated by the arrow), which corresponds to $>560 \mathrm{~kb}$ on the $P$. trichocarpa genome assembly.

Table 2 Chromosome XIX diversity statistics for individuals of $P$. alba with known sex, including expected $\left(H_{\mathrm{E}}\right)$ and observed $\left(H_{\mathrm{O}}\right)$ heterozygosity and inbreeding coefficients $\left(F_{\mathrm{IS}}\right)$ in each group and the female/male ratio of $H_{\mathrm{O}}$

\begin{tabular}{|c|c|c|c|c|c|c|c|c|c|}
\hline \multirow[t]{2}{*}{ Locus } & \multicolumn{4}{|c|}{ Females } & \multicolumn{4}{|c|}{ Males } & \multirow{2}{*}{$\begin{array}{c}\mathrm{H}_{O} \text { ratio } \\
\% / \widehat{0}\end{array}$} \\
\hline & $A$ & $H_{E}$ & $H_{O}$ & $F_{I S}$ & $A$ & $H_{E}$ & $H_{O}$ & $F_{I S}$ & \\
\hline Yin2 & 4 & 0.645 & 0.273 & 0.589 & 4 & 0.692 & 0.625 & 0.103 & 0.437 \\
\hline Yin1 & 3 & 0.301 & 0.333 & -0.114 & 3 & 0.492 & 0.625 & -0.296 & 0.533 \\
\hline Con03.1 & 3 & 0.610 & 0.909 & -0.527 & 3 & 0.689 & 1.000 & -0.539 & 0.909 \\
\hline Con49.1 & 5 & 0.667 & 0.500 & 0.258 & 4 & 0.592 & 0.625 & -0.061 & 0.800 \\
\hline Con 49.2 & 5 & 0.825 & 0.500 & 0.411 & 5 & 0.842 & 0.375 & $0.571^{\mathrm{a}}$ & 1.333 \\
\hline Con58.1 & 9 & 0.892 & 1.000 & -0.128 & 9 & 0.858 & 0.750 & 0.134 & 1.333 \\
\hline O206 & 2 & 0.290 & 0.333 & -0.158 & 2 & 0.325 & 0.375 & -0.167 & 0.888 \\
\hline $\mathrm{O} 276$ & 5 & 0.656 & 0.750 & -0.151 & 5 & 0.700 & 0.625 & 0.114 & 1.200 \\
\hline
\end{tabular}

a Significantly different from zero at the 0.05 level.

P. tremula (Table 1). A survey of trees with known sex indicated autosomal behavior of this chromosome: there were no consistent departures from random mating (measured via $F_{\text {IS }}$ ) in known females and males and no consistent differences in heterozygosity between known females and males (Table 2). LD on chromosome XIX extended over $>560 \mathrm{~kb}$ in natural populations of $P$. alba (Table 3), the species with the smaller effective population size, $N_{\mathrm{e}}$ (Lexer et al., 2005). The markers in LD corresponded to those loci with zero recombination in the interspecific $\mathrm{BC}_{1}$ (see Figure 1 and above). LD was also observed in the Swedish population of P. tremula, whereas no LD was detectable in P. tremula from the Eastern Alps (Table 3).

\section{Congruence between $\mathrm{BC}_{1}$ segregation patterns and} genomic divergence in natural populations

The increased introgression of $P$. tremula (=donor) alleles on chromosome $\mathrm{XIX}$ in the interspecific $\mathrm{BC}_{1}$
(Figure 2a) was mirrored by reduced interspecific divergence in natural populations when measured as $F_{\mathrm{ST}}$ (Figure $2 b$ ); the median of interspecific $F_{\mathrm{ST}}$ was far below the genome-wide expectation of 0.369 reported by Lexer et al. (2010). For comparison, no such reduction in interspecific divergence was seen on chromosome VI, consistent with normal segregation on this chromosome (Figure 2). A congruent pattern among chromosomes XIX and VI was recovered when Hedrick's (2005) $G^{\prime}$ ST was used as a measure of divergence $\left(G^{\prime}{ }_{S T}=0.556 \pm\right.$ 0.124 on chromosome XIX vs $0.898 \pm 0.049$ on chromosome VI, respectively). The results allowed us to compare introgression patterns seen in the $\mathrm{BC}_{1}$ and patterns of interspecific divergence observed in natural populations.

\section{Discussion}

\section{Segregation distortions favor rather than impede introgression in a controlled cross of $P$. tremula and} P. alba

Segregation distortions of genetic markers in interspecific crosses contain a wealth of information on the strength and genomic architecture of post-mating isolation between species (Fishman et al., 2001; Yin et al., 2004; Bouck et al., 2005; Sweigart et al., 2006; Lopez-Fernandez and Bolnick, 2007; Turelli and Moyle, 2007). This can point evolutionary biologists to genetic loci involved in speciation and to possible causes for asymmetries in reproductive barriers (Coyne and Orr, 2004; Turelli and Moyle, 2007) and breeders to genome regions that will resist introgression in marker-assisted breeding programs. Sometimes, however, the direction of segregation distortions in interspecific crosses also suggests the presence of mechanisms that favor rather than impede gene exchange (for example Tiffin et al., 2001; Yin et al., 
Table 3 Linkage disequilibrium (LD) among markers on chromosome XIX in natural populations of $P$. alba and P. tremula

\begin{tabular}{|c|c|c|c|c|c|}
\hline $\operatorname{Locus}^{\mathrm{a}}$ & Yin2 & Yin1 & Con49.2 & Con58.1 & O206 \\
\hline \multicolumn{6}{|c|}{ (a) P. alba/Austrian Danube valley } \\
\hline Yin2 (863 793) & - & $0.331^{*}$ & $0.154^{*}$ & $0.208^{*}$ & 0.658 \\
\hline Yin1 (873 555) & $0.000^{*}$ & - & 0.006 & 0.016 & 0.532 \\
\hline Con49.2 (1 296 123) & $0.000^{*}$ & 0.143 & - & 0.278 & 0.587 \\
\hline Con58.1 (1 426 114) & $0.000^{*}$ & 0.225 & 0.148 & - & 0.363 \\
\hline O206 (3 629 960) & 0.107 & 0.115 & 0.122 & 0.478 & - \\
\hline \multicolumn{6}{|c|}{ (b) P. alba/Hungarian Tisza valley } \\
\hline Yin2 (863 793) & - & 0.153 & 0.168 & 0.185 & $\mathrm{NC}$ \\
\hline Yin1 (873 555) & 0.487 & - & 0.152 & 0.032 & $\mathrm{NC}$ \\
\hline Con49.2 (1 296 123) & 0.008 & 0.134 & - & 0.008 & $\mathrm{NC}$ \\
\hline Con58.1 (1 426 114) & 0.441 & 0.160 & 0.165 & - & $\mathrm{NC}$ \\
\hline O206 (3 629 960) & $\mathrm{NC}$ & $\mathrm{NC}$ & NC & $\mathrm{NC}$ & NC \\
\hline \multicolumn{6}{|c|}{ (c) P. tremula/Eastern Alps } \\
\hline Yin2 (863 793) & - & 0.156 & 0.123 & 0.118 & 0.980 \\
\hline Yin1 (873 555) & 0.593 & - & 1.000 & 0.936 & 0.510 \\
\hline Con49.2 (1 296 123) & 1.000 & 0.121 & - & 0.271 & 0.903 \\
\hline Con58.1 (1 426 114) & 0.346 & 0.118 & 0.165 & - & 0.677 \\
\hline O206 (3 629 960) & 0.084 & 0.118 & 0.113 & 0.124 & - \\
\hline \multicolumn{6}{|l|}{ (d) P. tremula/Sweden } \\
\hline Yin2 (863 793) & - & 0.197 & 0.186 & 0.192 & 0.482 \\
\hline Yin1 (873 555) & 0.284 & - & 0.111 & 0.111 & 0.784 \\
\hline Con49.2 (1 296 123) & 0.034 & 0.166 & - & 0.010 & 0.007 \\
\hline Con58.1 (1 426 114) & 0.323 & 0.188 & 0.154 & - & 0.723 \\
\hline O206 (3 629 960) & 0.101 & 0.149 & 0.142 & 0.148 & - \\
\hline
\end{tabular}

Abbreviation: NC, not calculated.

Marker correlations are shown above the diagonal and exact $P$-values are shown below the diagonal. LD extending up to $562 \mathrm{~kb}$ (assuming $P$. trichocarpa map distances) is detectable in populations of $P$. alba ( $a$ and $b$ ), the species with the smaller effective population size $\left(N_{\mathrm{e}}\right)$, whereas LD is less readily detectable in populations of $P$. tremula (c and d).

Significant tests at the 0.05 level are indicated by bold type, and significant tests after Bonferroni correction are indicated by an asterisk.

The physical location of each marker in P. trichocarpa genome assembly v. 2 is shown in parentheses.

2004; Bouck et al., 2005), a pattern reminiscent of adaptive introgression. These cases are of great interest to our understanding of the genetics of porous species boundaries (Barton, 2001; Tiffin et al., 2001; Wu, 2001; Lexer and Widmer, 2008; Widmer et al., 2009), but understanding the causes of these patterns is not a trivial task.

Here, we uncovered extensive segregation distortions (20\% of polymorphic loci) in a controlled interspecific $\mathrm{BC}_{1}$ of $P$. alba and P. tremula, two naturally hybridizing, ecologically divergent forest trees, and almost all of these distortions (12 out of 13; >90\%) favored introgression of donor ( $P$. tremula) alleles into the heterospecific P. alba genomic background (Table 1). These allelic distortions manifested themselves also at the zygotic (=genotypic) level, and high levels of mortality (34\%) were observed during early diploid life stages of the progeny (first-year seedlings, a critical life stage in long-lived forest trees). Thus, the observed patterns are most easily explained by three non-exclusive hypotheses: (1) epistatic interactions between the hybridizing genomes (Coyne and Orr, 2004), (2) overdominance or heterozygote advantage in hybrids (Hartl and Clark, 1997), (3) cyto-nuclear coadaptation (Galloway and Fenster, 1999; Futuyma, 2009). The data allow us to address each of these hypotheses in turn.
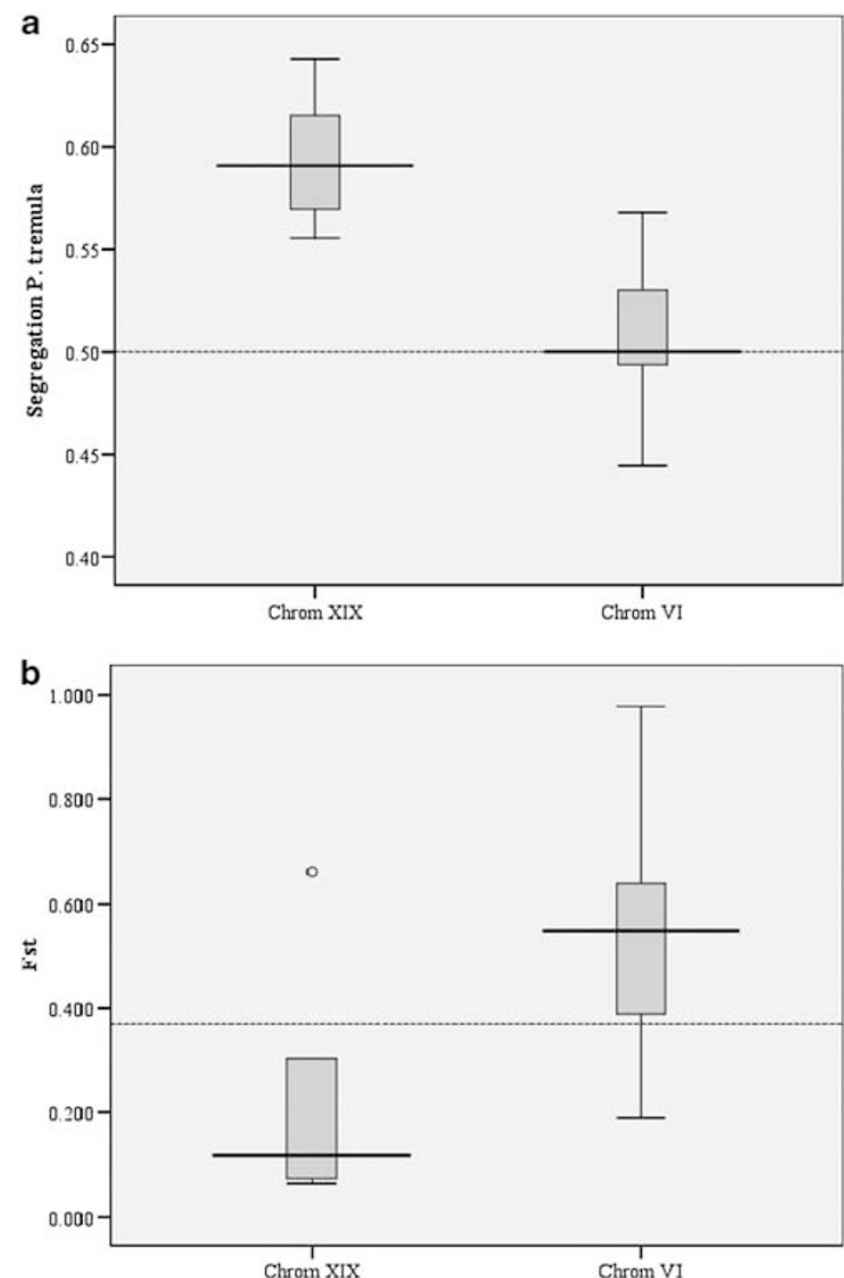

Figure 2 Box plots showing introgression and divergence of genetic markers on chromosomes VI and XIX relative to genome-wide expectations. (a) Segregation of $P$. tremula alleles in the interspecific $\mathrm{BC}_{1}$ (dotted line, Mendelian expectation of 0.5). (b) Interspecific divergence $\left(F_{\mathrm{ST}}\right)$ in natural populations (dotted line, genome-wide expectation of 0.369; Lexer et al., 2010). Increased introgression of $P$. tremula alleles in the controlled interspecific $\mathrm{BC}_{1}(\mathbf{a})$ and reduced interspecific divergence in natural populations (b) are visible for chromosome XIX.

Hypothesis 1, epistasis, refers to a central question in current speciation genetics, namely the relative role of Bateson-Dobzhansky-Muller incompatibilities vs other mechanisms in post-mating RI (Coyne and Orr, 2004; Turelli and Moyle, 2007). Evidence for epistasis comes from a recent analysis of genomic admixture in hybrid zones between these species: heterospecific genomic interactions clearly contribute to steep genomic clines in localities, where these species co-occur (Lexer et al., 2010). Nevertheless, epistasis alone is unlikely to generate the observed unidirectional bias in genotype frequencies observed here, consistently favoring alleles of the same species across multiple loci (Fishman et al., 2001). In fact, epistasis may be expected to produce the opposite pattern (a bias toward overrepresentation of $P$. alba alleles), so additional mechanisms must be invoked.

Hypothesis 2, heterozygote advantage (Hartl and Clark, 1997), is less frequently invoked in speciation genetic studies, but represents a mechanism of great interest in ecological and conservation genetics (Conner and Hartl, 
2004). Under this hypothesis, selection favoring interspecific heterozygotes will elevate the frequency of $P$. tremula donor alleles in the backcross. This hypothesis is supported by high mortality (34\%) during early life stages of the $\mathrm{BC}_{1}$ progeny and by the low level of heterozygosity of the $P$. alba backcross parent (only $38 \%$ ), compared with $67 \%$ of heterozygous loci in the $\mathrm{F}_{1}$ hybrid parent of the backcross. This suggests that increased heterozygosity due to introgression can ameliorate the negative effects of biparental inbreeding (that is of recessive deleterious alleles in homozygous state) in $P$. alba; biparental inbreeding in $P$. alba becomes apparent from the great magnitude of short-range kinship coefficients among individuals $\left(F_{\mathrm{ij}}\right)$ in recent studies of spatial genetic structure in this species (van Loo et al., 2008), and from the extra-ordinary clone sizes of genets of $P$. alba in Southern Europe (Brundu et al., 2008; González-Martínez and coworkers, unpublished data).

Hypothesis 3, increased introgression due to cytonuclear interactions (Galloway and Fenster, 1999; Tiffin et al., 2001; Futuyma, 2009), is equally supported by our data: our plastid DNA data indicate a $P$. tremula cytoplasm for the female $F_{1}$ hybrid parent of our interspecific $\mathrm{BC}_{1}$. Maternal inheritance of cytoplasmic genomes implies that all $\mathrm{BC}_{1}$ progeny will carry cytoplasmic genes from $P$. tremula, so nuclear $P$. tremula alleles segregating in the interspecific $B_{1}$ will be favored by selection in combination with the maternally derived conspecific cytoplasm present in each individual. In effect, cyto-nuclear coadaptation (or genomic conflict between heterospecific cyto-nuclear combinations) will 'pull' P. tremula nuclear alleles into a $P$. alba genomic background, resulting in a pattern that resembles, but should not be mistaken with adaptive introgression.

In the absence of reciprocal crosses, it is impossible to reject or accept either of these hypotheses with certainty. The present study was based on a single successful interspecific $\mathrm{BC}_{1}$ obtained by crossing an individual of $P$. alba with a natural $\mathrm{F}_{1}$ hybrid. A reciprocal crossing design (each species used as pollen and seed donor) would allow us to distinguish between cyto-nuclear and purely nuclear effects (Galloway and Fenster, 1999). While the absence of reciprocal crosses represents a caveat, congruent results (see below) from controlled progeny and natural populations indicate the generality of our findings beyond the successful cross used for segregation tests.

The well-documented selection episode in first-year seedlings and the clear consistency with genetic data for natural populations also allow us to interpret our results in the context of those obtained for many other groups of plants (Tiffin et al., 2001). Our results suggest that asymmmetries in post-mating barriers in these forest trees may result in introgression rather than the evolution of reinforcement upon secondary contact. In fact, two of the loci with significant overrepresentation of $P$. tremula alleles in the controlled cross (GCPM 1629 on chromosome 3 and ORPM 149 on chromosome 10; Table 1) are already known to exhibit greater than neutral introgression of $P$. tremula alleles in a wellstudied natural hybrid zone (Lexer et al., 2010). Of course, other mechanisms may be operating in parallel, slowing down introgression and strengthening the 'filter' to interspecific exchange (Coyne and Orr, 2004; Futuyma,
2009; Lexer et al., 2010). Thus, our study adds to the ongoing debate regarding the effects of asymmetric barriers on the evolutionary dynamics of species interactions upon secondary contact (Tiffin et al., 2001; Coyne and Orr, 2004; Lopez-Fernandez and Bolnick, 2007; Turelli and Moyle, 2007; Veltsos et al., 2008; Widmer et al., 2009).

The sex determination region of Populus is not protected from interspecific gene flow

Sex chromosomes (or sex determination regions more generally) have received considerable attention as hotspots' of genes or other genetic factors involved in species isolation (Qvarnström and Bailey, 2009). Particular attention has been paid to the mechanisms underlying 'Haldane's rule,' that is the observation that in hybrid zones between divergent populations or species, the heterogametic sex will often be rare, absent or sterile (reviewed by Coyne and Orr, 2004). More recently, the role of suppressed recombination on sex chromosomes has attracted much attention, triggered by the finding that reduced recombination greatly facilitates the accumulation of speciation genes on sex chromosomes (Qvarnström and Bailey, 2009). Both of these phenomena speak for an important role of sex chromosomes in speciation, and this should manifest itself in reduced introgression and increased divergence of these genome regions in recently diverged species (Nosil et al., 2009; Qvarnström and Bailey, 2009).

Our present results for the sex determination region of Populus are not consistent with this expectation. The proximal end of chromosome XIX exhibits suppressed recombination in our interspecific $\mathrm{BC}_{1}$ (Figure 1) and increased LD in natural populations (Table 3); note that LD normally decays within $1 \mathrm{~kb}$ in these species (Ingvarsson, 2008; Joseph and Lexer, 2008), and that patterns of LD vary with differences in effective population size $\left(N_{\mathrm{e}}\right)$ and metapopulation structure (Lexer et al., 2007). Still, interspecific divergence ( $F_{\mathrm{ST}}$ and $G^{\prime}{ }_{S T}$ ) of this region in natural populations is not greater than elsewhere in the genome (on the contrary, the opposite appears to be the case, see Figure 2 and Results). Hence, this genome region is not protected from interspecific gene flow. Consistent with this observation, genetic markers in this region display significant segregation distortion, consistently favoring $P$. tremula donor alleles in our interspecific $\mathrm{BC}_{1}$ toward $P$. alba (Table 1). These consistent but counter-intuitive results may be explained by characteristic features that distinguish the Populus sex determination region from other, better-studied sex chromosome systems.

First, the Populus sex determination region interrogated by our markers on chromosome XIX carries clusters of nucleotide-binding site-leucine rich repeat resistance (R-) genes (Yin et al., 2008). The fact that this important functional class of plant R-genes has been amplified in the Populus genome to form large clusters (Kohler et al., 2008) highlights their functional importance in these trees. If these nucleotide-binding siteleucine rich repeat genes are indeed under balancing selection as widely assumed for plant R-genes (Futuyma, 2009), then introgression may be favored by selection, especially in long-lived forest trees for which levels of standing variation are limited by notoriously low rates of 
molecular evolution per unit time (Petit and Hampe, 2006).

A possible alternative explanation for increased introgression on chromosome XIX is based on the known variability of sex determination systems in Populus. Whereas genomic data for the North American $P$. trichocarpa are suggestive of a $\mathrm{ZW}$ sex chromosome system involving a female-specific chromosomal segment located in the proximal end of chromosome XIX (Yin et al., 2008), genetic mapping of the sex locus in $P$. alba and in a cross $P$. tremula $\times$ Populus. tremuloides indicates the presence of at least two loci-controlling sex in a non-terminal position on this chromosome (Pakull et al., 2009; Paolucci et al., 2010). Interestingly, the sex locus maps to the female genetic map in P. alba (Paolucci et al., 2010) and to the male map in $P$. tremula $\times$ P. tremuloides (Pakull et al., 2009), consistent with the presence of two or more sex-controlling loci with different degrees of dominance. This would suggest that the Populus sex chromosome is at a very early step of its evolution, in which pairs or groups of sexually antagonistic mutations have accumulated, but full differentiation into heteromorphic sex chromosomes has not yet been achieved (Charlesworth et al., 2005). Our data are consistent with this hypothesis, since microsatellites in this region behave like codominant, autosomal markers and show no consistent pattern of reduced heterozygosity in either sex (Table 2). The apparent variation present in the poplar sex determination system (Yin et al., 2008; Pakull et al., 2009; Paolucci et al., 2010) provides a possible alternative explanation for increased introgression of the sex determination region (below).

\section{Conclusions and hypotheses for future work}

Our study demonstrates the value of experimental crosses involving natural hybrids of known genomic composition in understanding the genetics of species boundaries and barriers to introgression in Populus and other long-lived forest trees. Ongoing studies of natural hybrid zones between P. alba and P. tremula by our group have started to reveal patterns of genomic admixture and RI in multiple 'replicate' hybrid zone localities of these species across Europe (Lexer et al., 2007, 2010), which now provides a basis for picking natural hybrids and parental genotypes for controlled crossing experiments, such as those presented here. Experiments involving reciprocal crosses and both backcrossing directions (toward $P$. alba and $P$. tremula) will provide a more refined picture of post-mating and post-zygotic barriers to gene flow in these ecologically important trees.

With respect to the apparent lack of interspecific isolation of the sex determination region (above), a novel hypothesis has recently been put forward to explain the spread of novel sex-specific genome segments across hybrid zones (Veltsos et al., 2008). According to this hypothesis, a new chromosomal sex-determination system can spread across hybrid zones, even though it would normally be selected against within a single, isolated population (Pannell and Pujol, 2009). This apparently is made possible by the interplay between 'identity' disequilibria, commonly observed in hybrid zones, and the sexually antagonistic selection pressures affecting pairs or groups of sex-controlling loci (Veltsos et al., 2008; Pannell and Pujol, 2009).
Although Veltsos et al. (2008) modeled the specific case of a XY replacing a $X 0$ sex determination system, their model may be more widely applicable to other cases of asymmetric selection pressures in hybrid zones caused by dominance effects (that is 'dominance drive'; Mallet, 1986). The variable genetic architecture of sex determination in poplar (Yin et al., 2008; Pakull et al., 2009; Paolucci et al., 2010) could provide a suitable substrate for this type of process. Tests of the mode of spread and ecological impact of the sex determination region in hybrid zones between these species are currently underway.

\section{Conflict of interest}

The authors declare no conflict of interest.

\section{Acknowledgements}

We thank Joan Cottrell, Pär Ingvarsson, Berthold Heinze and Denes Bartha for help with obtaining samples from natural populations of poplars and aspens, the International Populus Genome Consortium (IPGC) for making genome assembly and primer sequences publicly available and Robyn Cowan and Dulcineia de Carvalho for help in the laboratory. CL's research on the evolutionary genomics of species barriers in Populus is supported by Grant no. NE/E016731/1 of the UK. Natural Environment Research Council (NERC) and Grant no. 31003A_127059 of the Swiss National Science Foundation (SNF).

\section{References}

Barton NH (2001). The role of hybridization in evolution. Mol Ecol 10: 551-568.

Bouck A, Peeler R, Arnold ML, Wessler SR (2005). Genetic mapping of species boundaries in Louisiana irises using IRRE retrotransposon display markers. Genetics 171: 1289-1303.

Brundu G, Lupi R, Zapelli I, Fossati T, Patrignani G, Camarda I et al. (2008). The origin of clonal diversity and structure of Populus alba in Sardinia: evidence from nuclear and plastid microsatellite markers. Ann Bot 102: 997-1006.

Charlesworth D, Charlesworth B, Marais G (2005). Steps in the evolution of heteromorphic sex chromosomes. Heredity 95: 118-128.

Conner JK, Hartl DL (2004). A Primer of Ecological Genetics. Sinauer Associates, Sunderland, USA.

Coyne JA, Orr HA (2004). Speciation. Sinauer Associates: Sunderland, MA.

De Carvalho D, Ingvarsson PK, Joseph J, Suter L, Sedivy C, Macaya-Sanz D et al. (2010). Admixture facilitates adaptation from standing variation in the European aspen (Populus tremula L.), a widespread forest tree. Mol Ecol 19: 1638-1650.

Emelianov I, Marec F, Mallet J (2004). Genomic evidence for divergence with gene flow in host races of the larch budmoth. Proc Biol Sci 271: 97-105.

Fishman L, Kelly AJ, Morgan E, Willis JH (2001). A genetic map in the Mimulus guttatus species complex reveals transmission ratio distortion due to heterospecific interactions. Genetics 159: 1701-1716.

Fussi B, Lexer C, Heinze B (2010). Phylogeography of Populus alba (L) and Populus tremula (L.) in Central Europe: secondary contact and hybridisation during recolonisation from disconnected refugia. Tree Genet Genomes 6: 439-450.

Futuyma DJ (2009). Evolution, 2nd edn. Sinauer Associates: Sunderland, MA. 
Galloway LF, Fenster CB (1999). The effect of nuclear and cytoplasmic genes on fitness and local adaptation in an annual legume, Chamaecrista fasciculata. Evolution 53: 1734-1743.

Goudet J (1995). FSTAT (version 1.2): a computer program to calculate F-statistics. J Hered 86: 485-486.

Hartl DL, Clark AG (1997). Principles of Population Genetics. Sinauer Associates: Sunderland, MA.

Hedrick PW (2005). A standardized genetic differentiation measure. Evolution 59: 1633-1638.

Ingvarsson PK (2008). Multilocus patterns of nucleotide polymorphism and the demographic history of Populus tremula. Genetics 180: 329-340.

Joseph JA, Lexer C (2008). A set of novel DNA polymorphisms within candidate genes potentially involved in ecological divergence between Populus alba and $P$. tremula, two hybridizing European forest trees. Mol Ecol Resources 8: 188-192.

Kohler A, Rinaldi C, Duplessis S, Baucher M, Geelen D, Duchaussoy F et al. (2008). Genome-wide identification of NBS resistance genes in Populus trichocarpa. Plant Mol Biol 66: 619-636.

Lexer C, Buerkle CA, Joseph JA, Heinze B, Fay MF (2007). Admixture in European Populus hybrid zones makes feasible the mapping of loci that contribute to reproductive isolation and trait differences. Heredity 98: 74-84.

Lexer C, Fay MF, Joseph JA, Nica MS, Heinze B (2005). Barrier to gene flow between two ecologically divergent Populus species, $P$ alba (white poplar) and $P$. tremula (European aspen): the role of ecology and life history in gene introgression. Mol Ecol 14: 1045-1057.

Lexer C, Joseph JA, van Loo M, Barbara T, Heinze B, Bartha D et al. (2010). Genomic admixture analysis in European Populus spp. reveals unexpected patterns of reproductive isolation and mating. Genetics 186: 699-712.

Lexer C, Joseph J, van Loo M, Prenner G, Heinze B, Chase MW et al. (2009). The use of digital image-based morphometrics to study the phenotypic mosaic in taxa with porous genomes. Taxon 58: 349-364.

Lexer C, Widmer A (2008). The genic view of plant speciation: recent progress and emerging questions. Philos Trans $R$ Soc Lond B Biol Sci 363: 3023-3036.

Lopez-Fernandez H, Bolnick DI (2007). What causes partial F1 hybrid viability? incomplete penetrance versus genetic variation. PLoS One 2: e1294.

Mallet J (1986). Hybrid zones of Heliconius butterflies in Panama and the stability and movement of warning color clines. Heredity 56: 191-202.

Nosil P, Funk DJ, Ortiz-Barrientos D (2009). Divergent selection and heterogeneous genomic divergence. Mol Ecol 18: 375-402.

Pakull B, Groppe K, Meyer M, Markussen T, Fladung M (2009). Genetic linkage mapping in aspen (Populus tremula L and Populus tremuloides Michx). Tree Genet Genomes 5: 505-515.

Pannell JR, Pujol B (2009). The paradoxical spread of a new $Y$ chromosome-a novel explanation. Trends Ecol Evol 24: 59-63.
Paolucci I, Gaudet M, Jorge V, Beritognolo I, Terzoli S, Kuzminsky E et al. (2010). Genetic linkage maps of Populus alba L. and comparative mapping analysis of sex determination across Populus species. Tree Genet Genomes 6: 863-875.

Petit RJ, Hampe A (2006). Some evolutionary consequences of being a tree. Annu Rev Ecol Evol Syst 37: 187-214.

Qvarnström A, Bailey RI (2009). Speciation through evolution of sex-linked genes. Heredity 102: 4-15.

Rousset F (2008). GENEPOP ' 007: a complete re-implementation of the GENEPOP software for Windows and Linux. Mol Ecol Resources 8: 103-106.

Smulders MJM, Van Der Schoot J, Arens P, Vosman B (2001). Trinucleotide repeat microsatellite markers for black poplar (Populus nigra L). Mol Ecol Notes 1: 188-190.

Sweigart AL, Fishman L, Willis JH (2006). A simple genetic incompatibility causes hybrid male sterility in Mimulus. Genetics 172: 2465-2479.

Tiffin P, Olson MS, Moyle LC (2001). Asymmetrical crossing barriers in angiosperms. Proc $R$ Soc Lond B 268: 861-867.

Turelli M, Moyle LC (2007). Asymmetric postmating isolation: Darwin's corollary to Haldane's rule. Genetics 176: 1059-1088.

Tuskan GA, DiFazio S, Jansson S, Bohlmann J, Grigoriev I, Hellsten U et al. (2006). The genome of black cottonwood, Populus trichocarpa (Torr. and Gray). Science 313: 1596-1604.

van der Schoot J, Pospiskova M, Vosman N, Smulders MJM (2000). Development and characterization of microsatellite markers in black poplar (Populus nigra L). Theor Appl Genet 101: 317-322.

van Loo M, Joseph JA, Heinze B, Fay MF, Lexer C (2008). Clonality and spatial genetic structure in Populus $x$ canescens and its sympatric backcross parent $P$ alba in a Central European hybrid zone. New Phytol 177: 506-516.

Veltsos P, Keller I, Nichols RA (2008). The inexorable spread of a newly arisen neo-Y chromosome. PLoS Genet 4: e1000082.

Widmer A, Lexer C, Cozzolino S (2009). Evolution of reproductive isolation in plants. Heredity 102: 31-38.

Woolbright SA, DiFazio SP, Yin T, Martinsen GD, Zhang X, Allan GJ et al. (2008). A dense linkage map of hybrid cottonwood (Populus fremontii $\times P$. angustifolia) contributes to long-term ecological research and comparison mapping in a model forest tree. Heredity 100: 59-70.

Wu C-I (2001). The genic view of the process of speciation. J Evol Biol 14: 851-865.

Yin TM, DiFazio SP, Gunter LE, Riemenschneider D, Tuskan GA (2004). Large-scale heterospecific segregation distortion in Populus revealed by a dense genetic map. Theor Appl Genet 109: 451-463.

Yin TM, DiFazio SP, Gunter LE, Zhang X, Sewell MM, Woolbright SA et al. (2008). Genome structure and emerging evidence of an incipient sex chromosome in Populus. Genome Res 18: 422-430.

Yin TM, Zhang XY, Gunter LE, Li SX, Wullschleger SD, Huang MR et al. (2009). Microsatellite primer resource for Populus developed from the mapped sequence scaffolds of the Nisqually-1 genome. New Phytol 181: 498-503.

Supplementary Information accompanies the paper on Heredity website (http://www.nature.com/hdy) 